\title{
Downlink and Uplink Message Size Impact on Round Trip Time Metric in Multi-Hop Wireless Mesh Networks
}

\author{
Youssra Chatei \\ Dept. Electronics, Informatics and Telecommunications \\ ENSAO, Mohammed I University \\ Oujda, Morocco \\ Maria Hammouti \\ Dept. Electronics, Informatics and Telecommunications \\ ENSAO, Mohammed I University \\ Oujda, Morocco
}

\author{
El Miloud Ar-reyouchi \\ Dept. Telecommunication and Computer Science \\ Abdelmalek Essaadi University \\ Tetouan, Morocco \\ Kamal Ghoumid \\ Dept. Electronics, Informatics and Telecommunications \\ ENSAO, Mohammed I University \\ Oujda, Morocco
}

\begin{abstract}
In this paper, the authors propose a novel real-time study metrics of Round Trip Time (RTT) for Multi-Hop Wireless Mesh Networks. They focus on real operational wireless networks with fixed nodes, such as industrial wireless networks. The main aim of the metric is to show the effect of the Downlink and Uplink message size (DMS and UMS) on RTT with and without Forward Error Correction (FEC), user data size without any headers, on RTT path between a source (Supervisory Control and Data Acquisition (SCADA) center) and a destination RTU (Remote Terminal Units). The metric assigns weights to links based on the RTT path of a packet size over the link path. They studied the performance of the metric by implementing it in three wireless scenarios consisting of 3,4 and 5 nodes; each node represents a wireless radio IP router. They find that in a multihops environment, the real-time metric clearly shows the impact of DMS, UMS and FEC on RTT by making judicious use of the number of the hops.
\end{abstract}

Keywords-RTT; Remote wireless communications; Wireless radio router; Wireless multi-hop networks; Average message size; FEC; $S C A D A$

\section{INTRODUCTION}

In this work, the authors consider the RTT as the time required for a packet to travel from the source (SCADA center) to the destination (remote RTU) and back again.

The SCADA center allows technical operation management for PV power plants on site. It permits us to visualize all measured values locally, in real time and in the event of a fault, reacts quickly and efficiently.

A remote terminal unit (RTU) is a multipurpose device used for remote monitoring and control of various devices and systems for automation.

In wireless network, the RTT is considered as an important measure in determining the accomplishment of a connection. RTT is effective and profitable in measuring the congestion window size and retransmission timeout of a connection [1] [2] [3] as well as the available bandwidth on an along path [4].
This information can help fixed, determine factors that limit data flow rates and cause congestion [5]. When a network link along the path is experienced, RTT can also aid efficient queue management and buffer provisioning. Moreover, RTT can be used to ameliorate node distribution in peer-to-peer and coverage networks [6].

With the wide range of RTU and PLC (Programmable Logic Controllers) currently on the market, SCADA system engineers [7] and decision makers face several challenges [8].

In basic SCADA architectures [9], information from a set of nodes (node-set) in wireless sensor networks (WSNs) [10], sensors or manual inputs are sent to PLCs or RTUs, which then send that information to computers with SCADA software. SCADA software analyzes and displays the data in order to help operators and other workers to reduce waste and improve efficiency in the manufacturing process.

The advent of Wireless Mesh Networking technology has introduced some very effective solutions for applications that previously required impractical and very expensive infrastructure. A Multi-Hop Wireless Mesh Networks [11] ,[12] can deliver large amounts of data and causes significant End-to-End delays across long distances using lot of nodes [13].

Wireless mesh networks also provide the added benefit of link redundancy for continued reliability across the infrastructure. Mesh networking helps to improve on the efficiency that Progressive Communications already delivers with custom SCADA and Digital Monitoring systems.

With Wireless Mesh Networks and SCADA monitoring, Progressive Communications can deliver solutions for applications such as:

1) Point-to-Multipoint wide area video surveillance

2) The peer-to-peer $(\mathrm{P} 2 \mathrm{P})$ renewable energy

3) Multipoint-to-Multipoint wide area video surveillance (law enforcement monitoring surveillance from their vehicles) 
4) Public Wi-Fi access systems for municipal parks and commons

5) Data gathering for wireless Electric / Water /Gas meters

6) Remote monitoring of analog systems (SCADA)

7) Remote monitoring of sub-stations (such as city-wide water pumps or another municipal infrastructure)

8) Multi-purpose wireless backbones (multiple systems utilizing the same available network).

FEC is a coding technology widely used in communication systems[14]. The principle is that the sender inserts some redundant data into its messages. This redundancy allows the receiver to detect and correct errors. The traditional FEC technique can increase the RTT efficiency in single-hop wireless networks such as WLAN or WiMAX networks. Using the FEC approach, the source encodes additional information together with the data before broadcast them to the receivers. If the amount of lost data is sufficiently small, a receiver can recover the lost data using some decoding schemes.

The industrial narrowband land mobile radio devices, as treated in this paper, have been the subject of European standard ETSI EN 300113 [15], ,[16]. The system functions on frequencies between $30 \mathrm{MHz}$ and $1 \mathrm{GHz}$, with channel separations of up to $50 \mathrm{kHz}$, and is designed for private, fixed, or mobile, radio packet switching networks.

The rest of the paper is organized as follows. The system model is introduced in Section II. The experimental setup is given in Section III. The description of the performance network and the metric results are presented in section IV. Finally, the authors conclude the paper and announce the future work in Section V.

\section{SYSTEM MODEL}

Let us consider the wireless network having $\mathrm{n}$ nodes as shown in Fig.1.

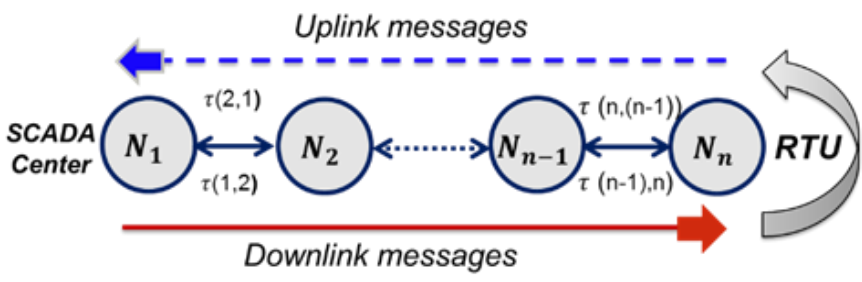

Fig. 1. Downlink and Uplink broadcast paths for each node

where, $n$ is the number of nodes presented in the respective RTT path, $\tau(1,2)$ is the time between the node $1 \& 2$ and depends upon the distance among them. The $\tau(i, j)$ is the time among the node $i$ and $j$ respectively.

Each downlink message is sent by the SCADA center network server to only one end device (RTU) and is relayed by a radio router. While end devices (RTUS) send Uplink messages to the network server relayed by one or many radio routers. Downlink and Uplink messages use the radio packet explicit mode in which any headers are included.
Consider the condition of having $n$ nodes in RTT path wich will be the maximum nodes in this Wireless Network. A worstcase application is constructed by the following path,

$$
\text { RTT path=Downlink RTT path + Uplink RTT path }
$$

So, the generalized equation for RTT time for the RTT path containing $\mathrm{n}$ nodes will be written as follows:

$$
\text { RTTpath }=\left(N_{1} \square \quad N_{2} \ldots . . \quad N_{n-1} \square \quad N_{n}\right)
$$

Where, the symboles $\quad \square$ or $\square \quad$ means the direction of the motion path of time that can takes a message to go from center to RTU and return to the center, through all specified intermediate nodes. RTTpath $\left(N_{i} \square \quad N_{j} \square \quad N_{l}\right)$ is the RTT among the node, ${ }_{i}, j$ and $l$ respectively, $(i, j, l \in\{1.2 \ldots n\})$.

The minimum number of routers required to form RTT path is three [1] and maximum routers in RTT paths should not be more than (n-1). The $\delta$ routers that build the RTT path for Wireless network are limited as following:

$$
3 \leq \delta \leq \mathrm{n}-1
$$

In router mode, the node works as a standard IP Router with two interfaces (Radio and Ethernet) and two COM port devices without any compromise. There is a sophisticated anticollision protocol on radio channel, where every single packet is acknowledged. Moreover, each unit can simultaneously work as a store-and-forward repeater.

Packet size is also influenced, to a modest degree, by the transmission error rate. If a link is noisy, it is possible to add a FEC that allows the receiver in many cases to figure out which bits are corrupted, and fix them. This has the effect of improving the bit error rate at a cost of reducing throughput. In order to involve many more bits than are needed for error detection, additionally, FEC can be applied. Typically, if a communications technology proves to have an unacceptably high bit-error rate (such as wireless, case of the experimental measurements),

If propagation delay of the message is considered negligible, the transmission time or transfer time of a message is expressed as:

$$
T_{t}=L / p D
$$

Where, $L$ is the message length (bits) is divided into $p$ packets transmitted on the different carriers at the same rate of $\mathrm{D}$ (bit/s).

If only one packet is transmitted in a time $\mathrm{L}$ becomes;

$$
L=m .8
$$

Where $m$ is the user data (bytes) transmitted without any headers (IP, TCP, UDP, ... (consider the longest possible reply from RT,U),

To minimize radio channel impairments, it is essential to use the FEC technique which is a very effective method. Therefore, the next step is to introduce an error-correcting 
code to the different next senarions. This generally reduces the "virtual" bit-error rate (that is, the error rate as corrected) to acceptable levels. The improvement comes at the expense of the data rate throughput:

$$
D=b \times F E C
$$

Where, $b$ is the modulation rate and FEC is the Forward Error Correction.

It apparent from this relation that the lower the FEC ratio, the better the error correction capability and correspondingly the lower the data rate. This implies an increase in the packet transmission time. Indeed, the packet transmission time through radio channel in miliseconds can be obtained from the packet size in bit (message $=$ one packet) and the data rate in bit/s as:

Testing Connection using TCP (Transmission Control Protocol) [17] and it is noted that initial header options were with 20 bytes, but after the first ACK the header options were with 12 bytes, so, it is necessary to add 12 bytes' in every TCP packet. (TCP packets include a 12 bytes' header).

Packet transmission time $t_{p}$ can be calculated as follows:

$$
t_{p}=(m+12) \cdot 8 /(b \cdot F E C)
$$

Where, $t_{p}(\mathrm{~ms})$ is the time needed for the packet transmission, with:

$$
\begin{array}{lll}
\mathrm{FEC}=1.00 & \text { if } & \mathrm{FEC}=\mathrm{Off} \\
\mathrm{FEC}=0.75 & \text { if } & \mathrm{FEC}=\mathrm{On}
\end{array}
$$

It is noted that a Modbus RTU over TCP is used. Simply put, this is a Modbus RTU message transmitted with a TCP/IP wrapper and sent over a network instead of serial lines. The Server does not have a SlaveID since it uses an IP Address instead.

Fig.2 shows different RTT path scenarios of downlink transmission message for SCADA center and for uplink transmission message at remote RTUs. The node $N_{1}$ can be considered as the base station (BS).

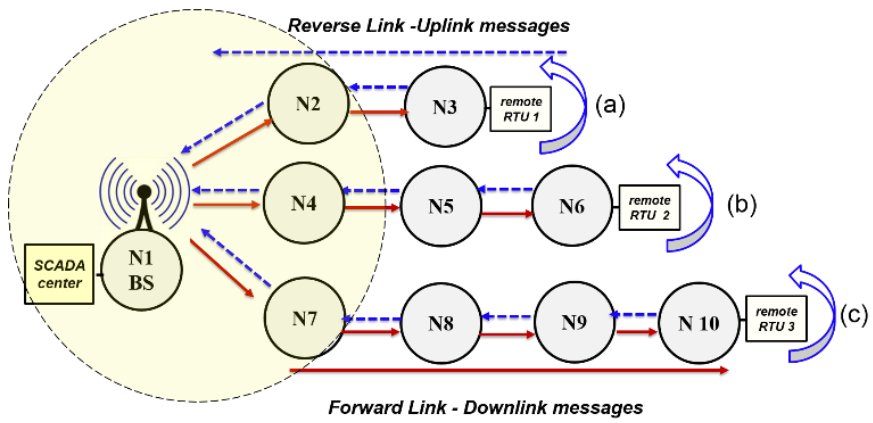

Fig. 2. Different RTT path scenarios of downlink and Uplink transmission message

In the topologies of the three scenarios, let us consider a graph including three, four and five nodes respectively. The RTT paths of network are calculated by using following equations:

1) Topology of the first scenario: Fig.2 (a)

$$
T_{R T T_{1}}=\tau(1,2)+\tau(2,3)+\tau(3,2)+\tau(2,1)
$$

2) Topology of the second scenario: Fig. $2(b)$

$T_{R T T_{2}}=\tau(1,4)+\tau(4,5)+\tau(5,6)+\tau(6,5)+\tau(5,4)+\tau(4,1)(9)$

3) Topology of the third scenario: Fig. 2 (c)

$$
\begin{aligned}
& T_{R T T_{2}}=\tau(1,7)+\tau(7,8)+\tau(8,9)+\tau(9,10)+\tau(10,9)+\tau(9,8)+ \\
& \tau(8,7)+\tau(7,1)
\end{aligned}
$$

The center broadcasts request and the RTUs 1, 2 and 3 generate the response and send it out to their respective nodes.

In this paper, through these metrics application, the frequency band $(160 \mathrm{MHz})$ is the best choice when covering a hilly region and repeaters are not an option. The only frequency of the set of options, which can possibly make it to a distant valley, $20 \mathrm{~km}$, Line of sight (LoS) as propagation mode, from the nearest point-of-presence, it can reach a ship $100 \mathrm{~km}$ from the shore base using radio router as the transmission medium with Simple Network Management Protocol (SNMP). Consequently, this frequency band is suitable for low speeds using robust modulation techniques only, and even then, somewhat lower long-term communication reliability has to be acceptable for this metric application.

\section{EXPERIMENTAL SETUP}

The system consists of two parts, related hardware and management software. The system hardware is divided into onsite data monitoring unit, wireless transmission unit and monitoring center. The software adopts decentralized collection and centralized control management model, providing users basic information management, real-time monitoring, fault alarm, fault records, maintenance reminder, maintenance records, failure statistics, bulletin boards, industry news and so on.

The equipment's, including computer software used, are:

- The SunSet E20c provides full transmission testing over $2.048 \mathrm{Mbit} / \mathrm{s}$ and V-series Datacom interfaces. It verifies Datacom circuits by monitoring the received data, control leads, and physical layer results. It also tests frame relay circuits, PING testing, stress testing, and statistics.

- Ten radio routers (use the frequency band $160 \mathrm{MHz}$ ) which are characterized by the SNMP (Simple Network Management Protocol) that will support the base MIB (Management Information Base).

- Omnidirectional antenna KA160.3 which is designed for base radio stations working in bands of 158-174 $\mathrm{MHz}$ The antenna, used in our application, has an omnidirectional radiation pattern with the gain of $3 \mathrm{~dB}$ and is adapted for the top-mounting. The values received at the level of each site vary between 38 and $70 \mathrm{~dB} \mu \mathrm{V}$.

- The output power of each radio router varies between 0.1 and 2 watts. 
The Processing time is the time for the RTUs / SCADA devices to process queries; it has the same value and Interface speed, in either a center or remote, which is $20 \mathrm{msec}$ and Ethernet-TCP/IP respectively.

The RTT measurement is affected by various parameters of the wireless network. The following Table I lists conditions selected.

TABLE. I. CONDITIONS SELECTED

\begin{tabular}{|l|l|}
\hline Factors Affecting RTT measurement & \multicolumn{1}{|c|}{ Condition Chosen } \\
\hline Total node Number of sites & 10 \\
\hline Average hops per path to remote & $4-6-8$ \\
\hline Operating Mode & Radio Router \\
\hline Modulation rate [kbps] & 16DEQAM (166.67) \\
\hline ACK: Acknowledgement & On \\
\hline FEC: Forward Error Correction & with $(\mathrm{FEC}=3 / 4) /$ without \\
\hline Processing time & $20 \mathrm{~ms}$ \\
\hline
\end{tabular}

\section{RESULTS}

This section describes the experiments results (see Fig. 2). The presented measurements show, firstly, how RTT (between source and destination) varies depending on the DMS (User data size without any headers) for several hops and UMS (500 Bytes, 1000 Bytes and 1500 Bytes) of wireless links, then their performances, of a variety of multi-hop wireless mesh networks to fixed value of UMS metric, are compared in various conditions.

The authors begin by comparing the performance of RTT to DMS as well as basic shortest-path routing using only three nodes per RTT path, 3 nodes and 4 Hops, (see Fig. 2):

$$
\operatorname{RTT}\left(N_{1} \square \quad N_{2} \square \quad N_{3}\right)
$$

Then for four nodes per RTT path (4 nodes and 6 Hops):

$$
R T T\left(N_{1} \square \quad N_{4} \square \quad N_{5} \square \quad N_{6}\right)
$$

Finally, the RTT path with five nodes (5 nodes and 8 Hops) is:

$$
R T T\left(N_{1} \square \quad N_{7} \square \quad N_{8} \square \quad N_{9} \square \quad N_{10}\right)
$$

Next, measurements results are obtained by selecting $\mathrm{UMS}=500,1000$ and 1500 Bytes and the performance of RTT in this tree path routing is compared.

Various measurements are effectuated, in comparison to different values of packet length. Each router units' module may support up to 1500 bytes (User data size without any headers (IP, TCP, UDP ...) of RF payload.

\section{A. RTT for multi-hop wireless path}

Fig.3 and Fig.4 show the RTT versus number of hops depending of different DMS/UMS and with or without FEC.
The results of the ping RTT, for multihop connections with all wireless hops (at the four, six, and eight hops) and with /without FEC, are showed in Fig.3 and Fig.4.

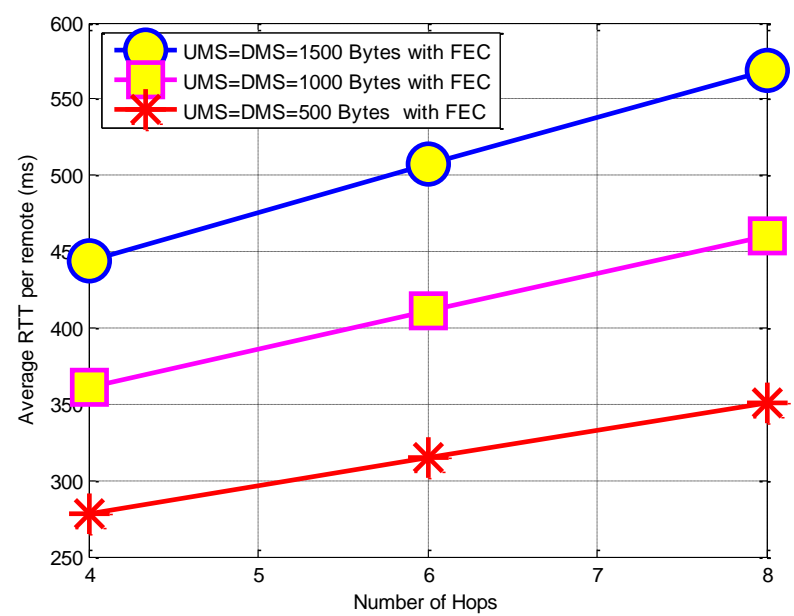

Fig. 3. The ping RTT for multihop connections with all wireless hops (at the four, six, and eight hops) and with FEC

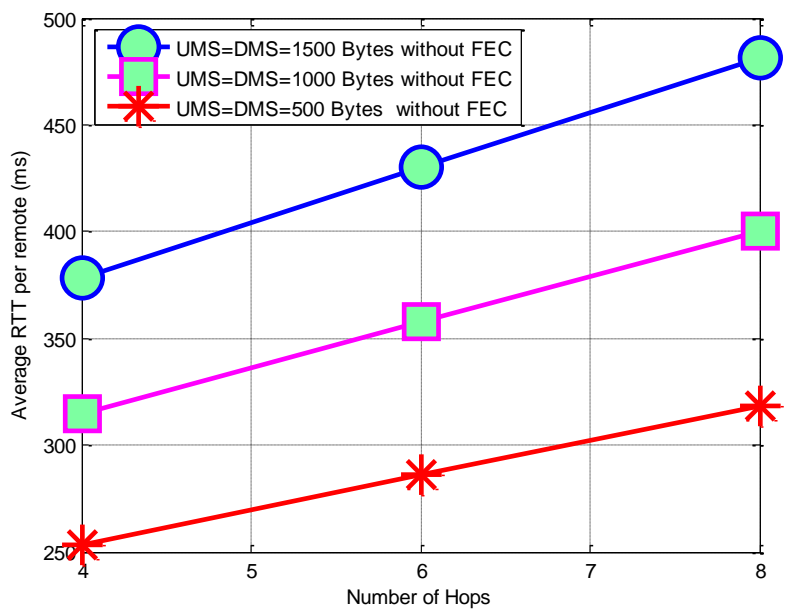

Fig. 4. The ping RTT for multihop connections with all wireless hops (at the four, six, and eight hops) and without FEC

As it can be seen in Fig.3 and Fig.4, both the number of hops and UMS/DMS can affect the RTT. In the two cases with / without FEC, the RTT increases when the number of hops or the UMS/DMS increases. Another important point obtained from these results is that the RTT measurements, by fixing the value of UMS=DMS at 1500,1000 and 500 bytes, increase of the same value from 4 hops to 6 hops and from 6 to 8 hops as illustrated in Table II.

TABLE. II. INCREASE OF RTT VALUES

\begin{tabular}{|c|l|l|l|l|l|l|}
\hline & & \multicolumn{3}{|l|}{ With FEC } & \multicolumn{3}{l|}{ Without FEC } \\
\hline UMS=DMS & $\mathbf{5 0 0}$ & $\mathbf{1 0 0 0}$ & $\mathbf{1 5 0 0}$ & $\mathbf{5 0 0}$ & $\mathbf{1 0 0 0}$ & $\mathbf{1 5 0 0}$ \\
\hline $4 \square \quad 6 \square \quad 8$ & 37 & 49 & 62 & 32 & 42 & 52 \\
\hline
\end{tabular}

In order to avoid reception overcharge with the SCADA communication protocol, a packet is a block of data with length 
that can vary between 0 to 1500 bytes. In this case, the shape of RTT curve is showed as a function of message size and the results are illustrated in Figs.5-10, for both situations with and without FEC, as described in the following three scenarios.

\section{B. Average RTT per remote: First scenario}

The first scenario is considered as shown in Fig. 2(a). It is worth mentioning that the direct transmission is considered between the SCADA center and the destination RTU1 using three nodes (radio router). Each node in Wireless Network is defined for a selected RTT path by configuring them, with SCADA center and RTU1, using the protocol SCADA software. The messages are relayed and RTT path selected here, as in (11), is:

$$
\operatorname{RTT}\left(N_{1} \square \quad N_{2} \square \quad N_{3}\right)
$$

Fig.5 and Fig.6 show the results of a representative first scenario practice with and without FEC respectively.

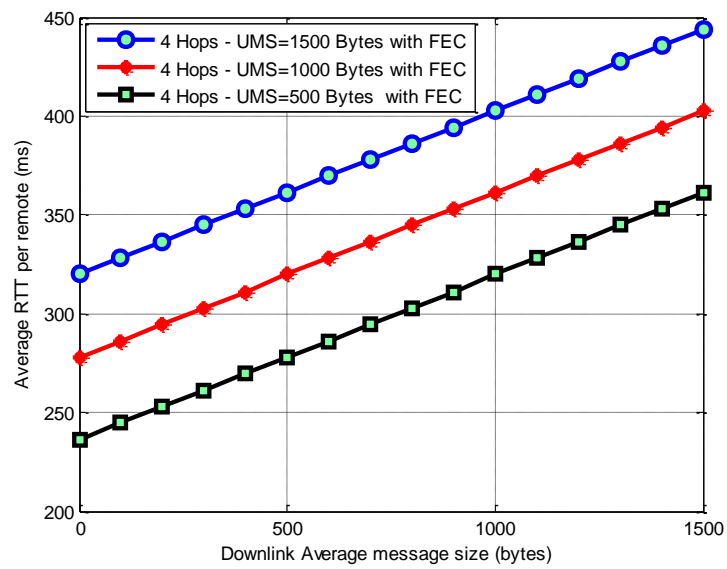

Fig. 5. Average RTT vs DMS: 4 Hops with FEC and different values of UMS

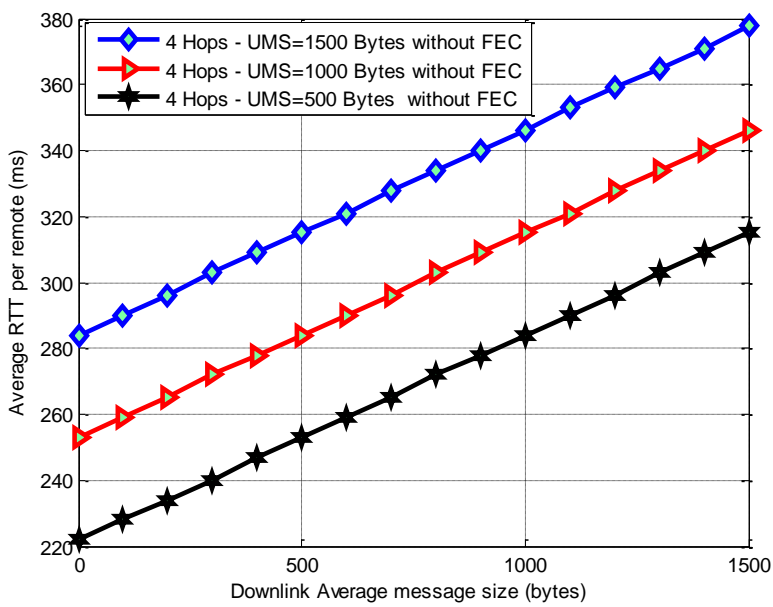

Fig. 6. Average RTT vs DMS: 4 Hops without FEC and different values of UMS

This experimental trial is repeated ten times. Furthermore, it was conducted to have a performance baseline for wireless networks.
The results in Fig.5 and Fig.6 shows that the RTT is increased when the DMS increases. The increase of UMS can also promote a slight increase in RTT. On the other hand, one can see from Fig.6 that the introduction of FEC can significantly increase the RTT.

For a fixed value of UMS $=1000$ Bytes and ranging DMS from 0 up to 1500 Bytes and without FEC, the RTT for 4 hops increases to $93 \mathrm{msec}$, whereas for $\mathrm{FEC}=3 / 4 \mathrm{RTT}$ increases to $125 \mathrm{msec}$.

\section{Average RTT per remote: Second scenario}

In Fig.7 and Fig.8, the topology showed in Fig.2 (b) is well respected. SCADA center unit, configured as a source, sends packets to destination RTU2 and comes back to (BS) then calculates the RTT. The direct transmission between the SCADA center and the destination RTU 2 is respected using four nodes (Router). The RTT path, as in (12), is:

$$
R T T\left(N_{1} \square \quad N_{4} \square \quad N_{5} \square \quad N_{6}\right)
$$

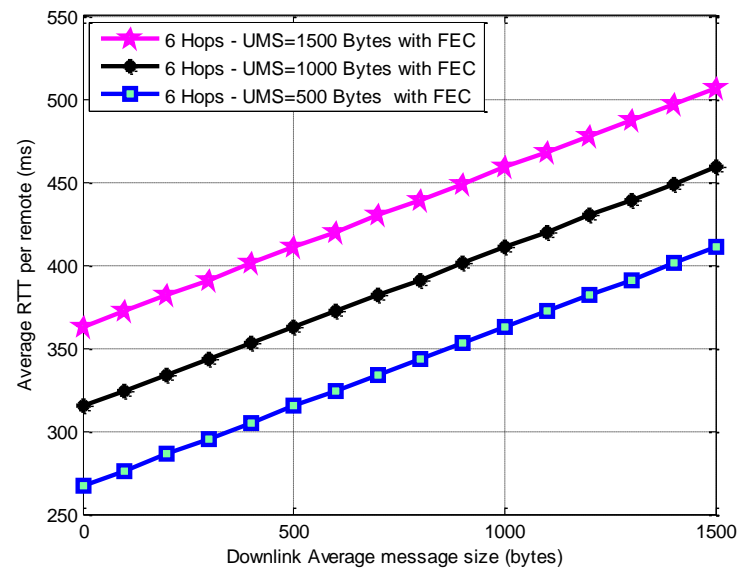

Fig. 7. Average RTT vs DMS: 6 Hops with FEC and different values of UMS

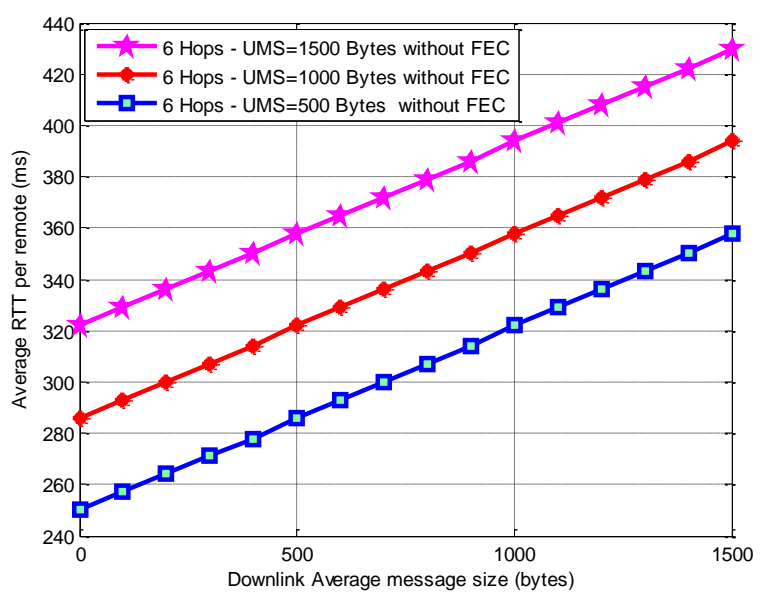

Fig. 8. Average RTT vs DMS: 6 Hops without FEC and different values of UMS

According to the results shown in Fig.7 and Fig.8, it is possible to observe that the RTT increases linearly as the DMS 
increases. The increase of UMS can also promote a significant increase in RTT. In the same way, as previously discussed, the introduction of FEC can significantly affect the RTT, the results show that FEC can indeed increase the RTT.

For a fixed value of UMS $=1000$ Bytes and ranging DMS from 0 up to 1500 Bytes, without FEC, the RTT for 6 hops increases to $108 \mathrm{msec}$. Whereas for $\mathrm{FEC}=3 / 4$, RTT increases to $144 \mathrm{msec}$.

\section{Average RTT per remote: Third scenario}

In Fig.9 and Fig.10, the network topology in Fig.2 (c) is fully considered where the messages are transmitted from the (BS) to the RTU 3 and return to the (BS). The messages are relayed using the following RTT path, as in (13):

$$
\operatorname{RTT}\left(N_{1} \square \quad N_{7} \square \quad N_{8} \square \quad N_{9} \square \quad N_{10}\right)
$$

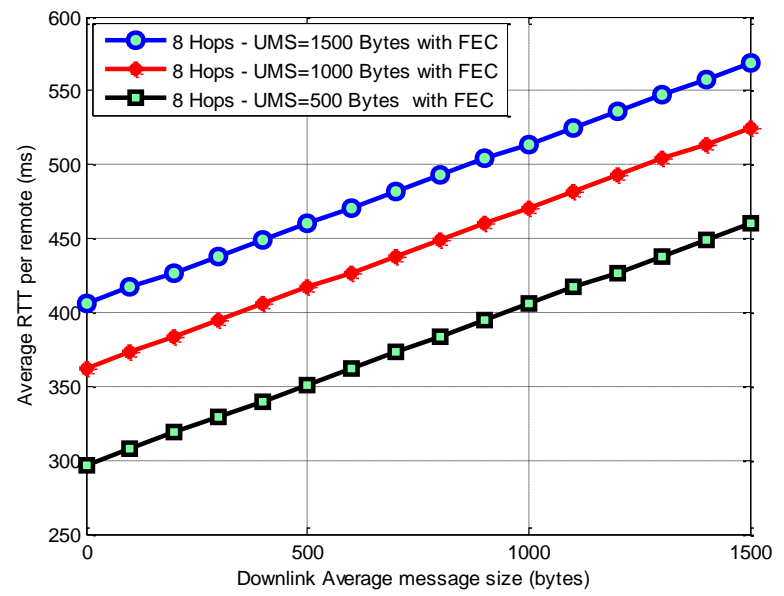

Fig. 9. Average RTT vs DMS: 8 Hops with FEC and different values of UMS

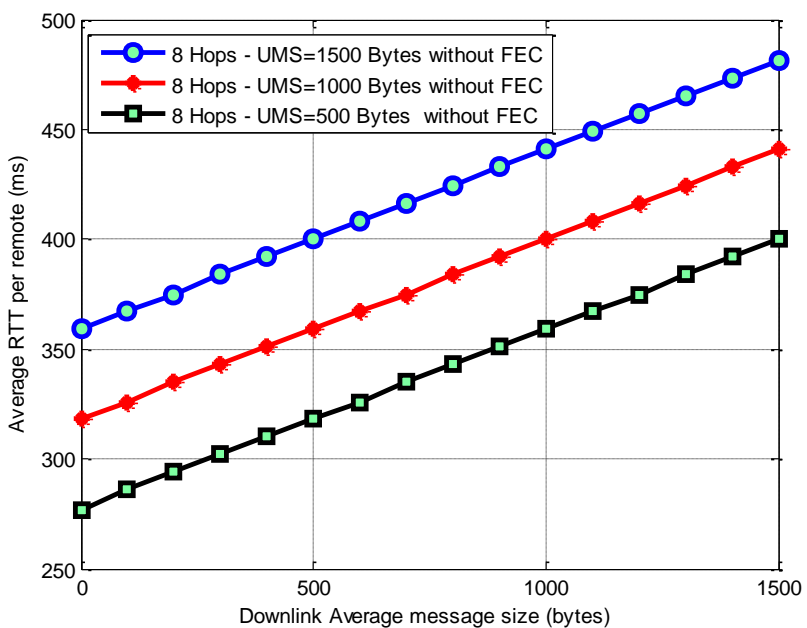

Fig. 10. Average RTT vs DMS: 8 Hops without FEC and different values of UMS
Similar to what happen in figs. 5-8, one can observe from the Fig. 9 and Fig.10 for a large number of hops ( 8 hops) that, depending of the value of UMS, the RTT increases linearly when the DMS increases.

Once again, it can be noted that for a fixed value of UMS= 1000 Bytes and ranging DMS from 0 up to 1500 Bytes and without FEC, the RTT, for 8 hops, increases to $123 \mathrm{msec}$, whereas for $\mathrm{FEC}=3 / 4$ it increases to $163 \mathrm{msec}$.

\section{E. Comparison of Results}

Fig.11 shows the comparison of results obtained by considering the first scenario for different UMS and DMS values and with/without FEC.

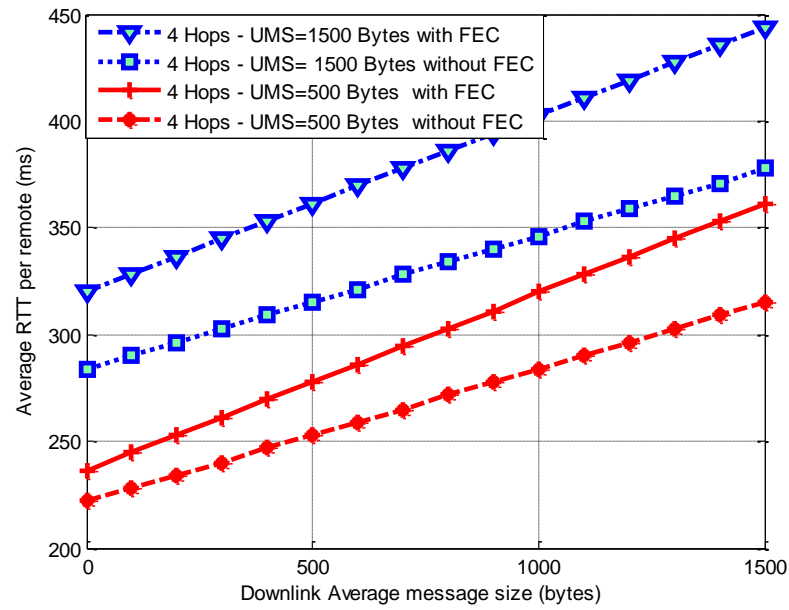

Fig. 11. The comparison of results obtained by considering a first scenario for different values of UMS and with/without FEC

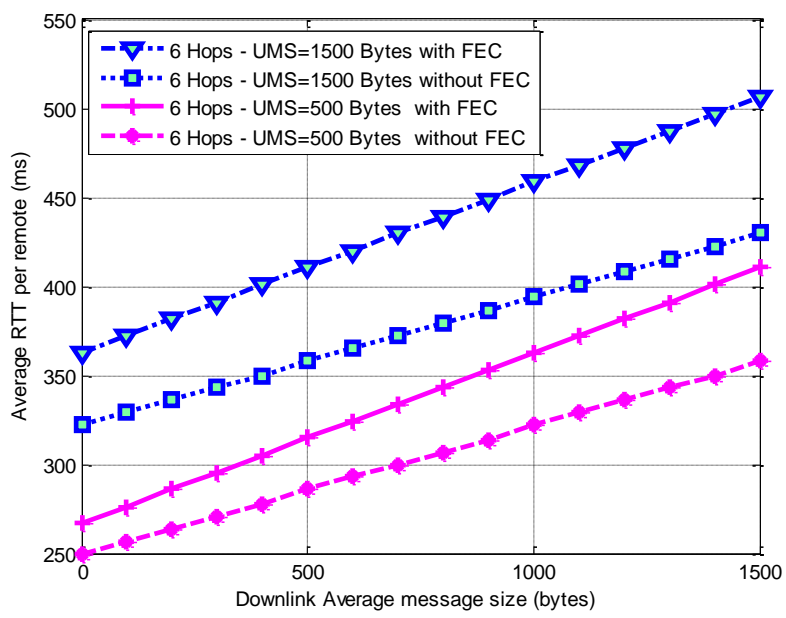

Fig. 12. The comparison of results obtained by considering a second scenario for different values of UMS and with/without FEC 


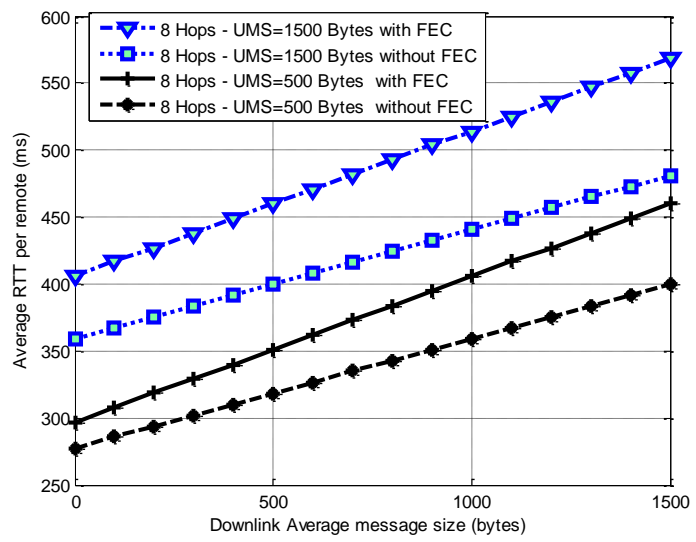

Fig. 13. The comparison of results obtained by considering a third scenario for different values of UMS and with/without FEC

The results in Fig.11 shows that the using different Downlink Average Message Size (bytes), Uplink Average Message Size (bytes) and FEC, in multi-hop wireless networks, have a significant effect on the Round-Trip Time (RTT) metric. We repeated the previous experiments with six and eight hops (second and third scenario) network respectively we obtained the same observations as illustrated in Fig 12 and Fig.13.

\section{CONCLUSIONS}

This paper presents a new method for measuring RTT giving a quick performance overview based on several basic parameters.

In light of the results obtained in real-time study metrics, it can therefore be concluded that RTT depends on various parameters such as the number of hops, DMS, UMS and FEC. Based on the results found, the most important concluding notes can be seen in the following:

- DMS has an effect on RTT measurements, when the DMS increases, the RTT also increases linearly in RTT path. Hence linear relationship exists between RTT and router node DMS in multi-hop wireless mesh networks.

- The offset between RTT, with and without FEC of different hop varieties, increases as the Downlink and Uplink message size increases.

- The UMS is another important factor that can affect the RTT measurements; it was observed that RTT increases as the UMS augments.

- Furthermore, it is observed that the coding technique Rate $(\mathrm{FEC}=3 / 4)$ provokes a further increase in RTT and the practical implementation of UMS, nodes and FEC, increase RTT without any influence on the linear relationship between RTT and router node DMS.

The offset between RTT, with and without FEC of different hop varieties, increases as the Downlink and Uplink message size increases.so, for relatively high error rates, it turns out to be better to send smaller packets, because when an error does occur then the entire packet containing it is lost.

\section{REFERENCES}

[1] El Miloud Ar-Reyouchi, Kamal Ghoumid, Koutaiba Ameziane, and Otman El Mrabet, "Performance Analysis of Round Trip Time in Narrowband RF Networks For Remote Wireless Communications", International Journal of Computer Science \& Information Technology (IJCSIT),5(5), pp.1-20, October 2013.

[2] Dalal, P., Sarkar, M., Kothari, N., and Dasgupta, K. ” Refining TCP's RTT dependent mechanism by utilizing link retransmission delay measurement in Wireless LAN”. Int J Commun Syst, 30,(5), 20170.

[3] El Miloud Ar Reyouchi, Kamal Ghoumid, Koutaiba Ameziane, Otman El Mrabet, Slimane Mekaoui, "Performance Analysis of Round Trip Delay Time in Practical Wireless Network for Telemanagement", International Journal of Electrical, Computer, Energetic, Electronic and Communication Engineering, Waset, 7(11), pp.1413-1419, 2013.

[4] El Miloud Ar-reyouchi , Koutaiba Ameziane, Otman. El Mrabet, Kamal Ghoumid, "The potentials of Network Coding for improvement of Round Trip Time in wireless Narrowband RF communications", Multimedia Computing and Systems (ICMCS), International Conference on, pp.765-770, 2014.

[5] Jain, M., Dovrolis, C. "End-to-end available bandwidth: measurement methodology, dynamics, and relation with tcp throughput". In: SIGCOMM, ACM, 2002.

[6] Zhang, Y., Breslau, L., Paxson, V., Shenker, S.2002. "On the characteristics and origins of Internet flow rates". In: SIGCOMM, ACM, 2002.

[7] Ratnasamy, S., Handley, M., Karp, R., Shenker, S. "Topologicallyaware overlay construction and server selection". In: INFOCOM, IEEE, 2002.

[8] Peter King "SCADA Systems - Looking Ahead"Control Microsystems White Paper, August 2005.

[9] STOUFFER, K., FALCO, J., AND KENT, K. Guide to supervisory control and data acquisition (scada) and industrial control systems security. Sp800-82, NIST, September 2006.

[10] K. Gowri Shankar "Control of Boiler Operation Using PLC- SCADA", International Multi Conference of Engineers and Computer Scientists, Vol II, IMECS , Hong Kong, March 19-21, 2008.

[11] R. Baumann, S. Heimlicher, V. Lenders and M. May, "Routing Packets into Wireless Mesh Networks," Third IEEE International Conference on Wireless and Mobile Computing, Networking and Communications (WiMob 2007), White Plains, NY,pp. 38-38.2007. doi: 10.1109/WIMOB.2007.

[12] Richard Draves, Jitendra Padhye Brian Zill, "Routing in multi-radio, multi-hop wireless mesh networks" Proceeding MobiCom '04 Proceedings of the 10th annual international conference on Mobile computing and networking Philadelphia, PA, USA - September 26 October 01 pp. 114-128, 2004

[13] R. Draves, J. Padhye, and B. Zill, "Routing in Multi-Radio, Multi-Hop Wireless Mesh Networks," ACM Annual Int'l. Conf. Mobile Comp. and Net. (MOBICOM), pp. 114-128, 2004.

[14] El Miloud Ar-Reyouchi,Youssra Chatei,Kamal Ghoumid,Ahmed Lichioui"'The Powerful Combined Effect of Forward Error Correction and Automatic Repeat Request to Improve the Reliability in the Wireless Communications", International Conference on Computational Science and Computational Intelligence (CSCI) Las Vegas, NV, USA Dec. , 2015,pp: 691-696, 2015.

[15] ETSI EN 300 113-1 V1.6.2 (2009-11), Electromagnetic compatibility and Radio spectrum Matters (ERM), Part 1: Technical characteristics and methods of measurement. European Standard. ETSI, 11/2009.

[16] ETSI EN 302561 V1.2.1 (2009-12), Electromagnetic compatibility and Radio spectrum Matters (ERM), Land Mobile Service; Radio Equipment using constant or non-constant envelope modulation operating in a channel bandwidth of $25 \mathrm{kHz}, 50 \mathrm{kHz}, 100 \mathrm{kHz}$ or $150 \mathrm{kHz}$; Harmonized EN covering essential requirements of article 3.2 of the R\&TTE Directive. European Standard. ETSI, 12/2009.

[17] Mohammad Shorfuzzaman, Mehedi Masud and Md. Mahfuzur Rahman, "Characterizing End-to-End Delay Performance of Randomized TCP Using an Analytical Model" International Journal of Advanced Computer Science and Applications(IJACSA), 7(3), 2016. 